Revue des patrimoines

$40 \mid 2019$

Lits historiques. Première anthologie des lits européens du XVe au XIXe siècle

\title{
Le lit de Napoléon et de Louis XVIII à Saint-Cloud retrouvé
}

The Bed of Napoleon I and of Louis XVIII found again at Saint-Cloud

\section{Renaud Serrette}

\section{(2) OpenEdition}

\section{Journals}

Édition électronique

URL : http://journals.openedition.org/insitu/23885

DOI : $10.4000 /$ insitu. 23885

ISSN : 1630-7305

Éditeur

Ministère de la Culture

Référence électronique

Renaud Serrette, "Le lit de Napoléon et de Louis XVIII à Saint-Cloud retrouvé », In Situ [En ligne], 40 I

2019, mis en ligne le 18 septembre 2019, consulté le 04 octobre 2019. URL : http://

journals.openedition.org/insitu/23885; DOI : 10.4000/insitu.23885

Ce document a été généré automatiquement le 4 octobre 2019.

\section{cc) () $९$}

In Situ Revues des patrimoines est mis à disposition selon les termes de la licence Creative Commons Attribution - Pas d'Utilisation Commerciale - Pas de Modification 4.0 International. 


\section{Le lit de Napoléon et de Louis XVIII à Saint-Cloud retrouvé}

The Bed of Napoleon I and of Louis XVIII found again at Saint-Cloud

\section{Renaud Serrette}

L'auteur tient à remercier pour leur aide Philippe Le Pareux, professeur agrégé d'histoire, JeanJacques Gautier, Emmanuelle Federspiel et Marie-Amélie Tharaud, inspecteurs au Mobilier national, Jean Vittet, conservateur en chef au château de Fontainebleau, Isabelle Vétois, conservatrice en chef au musée national des châteaux de Malmaison et de Bois-Préau, Judith Kagan, cheffe du bureau de la Conservation du patrimoine mobilier et instrumental, Isabelle de Gourcuff, administratrice générale du château de Fontainebleau, Anne Dion, conservatrice en chef au département des Objets d'art au musée du Louvre.

1 En 1983, un très beau lit en bois doré d'époque Consulat était proposé aux enchères à Drouot $^{1}$ (fig. 1). Le catalogue de vente précisait bien l'existence de "numéros du Mobilier national " mais on les considéra alors comme étant ceux du palais des Tuileries. Préempté par le Centre des monuments nationaux (CMN), on constata rapidement qu'aucun château parmi la centaine confiée à sa gestion ne présentait un cadre adapté pour présenter ce meuble 2 . Il fut donc envoyé en 1990 dans les réserves du château de Bouges (Indre-et-Loire) où il finit par être oublié dans les entresols puis dans les écuries. Or, un examen de ses marques a récemment permis de révéler les origines prestigieuses et l'histoire remarquable de ce meuble. 
Figure 1

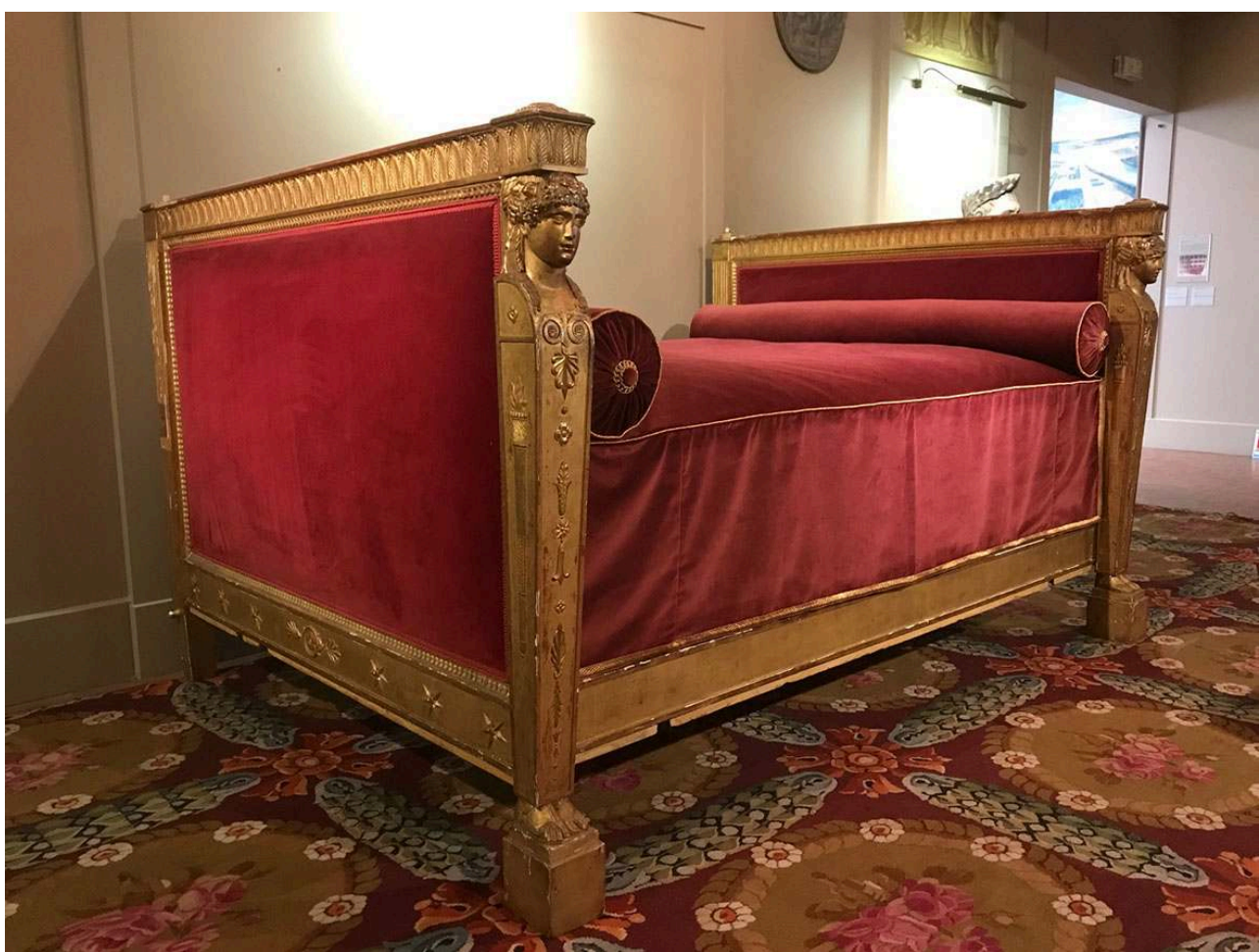

Attribué à Jacob Frères, lit en chaire à prêcher de Napoléon et de Louis XVIII à Saint-Cloud, vers 1799, bois doré. Centre des monuments nationaux, Domaine national de Saint-Cloud, BOU1983101619.

Phot. Renaud Serrette. (c) Renaud Serrette.

\section{Un lit d'époque Consulat}

Le lit se compose d'une seule couchette en bois doré, le ciel étant perdu. Les montants arrière, de même hauteur que ceux de devant, sont simplement cannelés et rudentés, et étaient encore, au moment de son acquisition, surmontés de vases tournés en forme d'urne $^{3}$. Les sommets des deux chevets sont finement sculptés d'une frise de feuilles d'eau et de tigettes de lilas tandis que les traverses latérales sont ornées de rosettes encadrées de palmettes et d'étoiles. Les montants avant sont sculptés de figures en gaine sommées de têtes de jeunes filles couronnées de fleurs avec des rubans flottant sur le cou (fig. 2). Les gaines sont sculptées sur la face principale de palmettes, rosettes et patères, et sur les côtés, de torches allumées. Elles se terminent par des pieds sur un socle. Le motif de la figure en gaine ornant les montants avant, déjà employé à la fin du XVIII ${ }^{e}$ siècle, connut un grand succès au début du XIX ${ }^{e}$ siècle. 
Figure 2

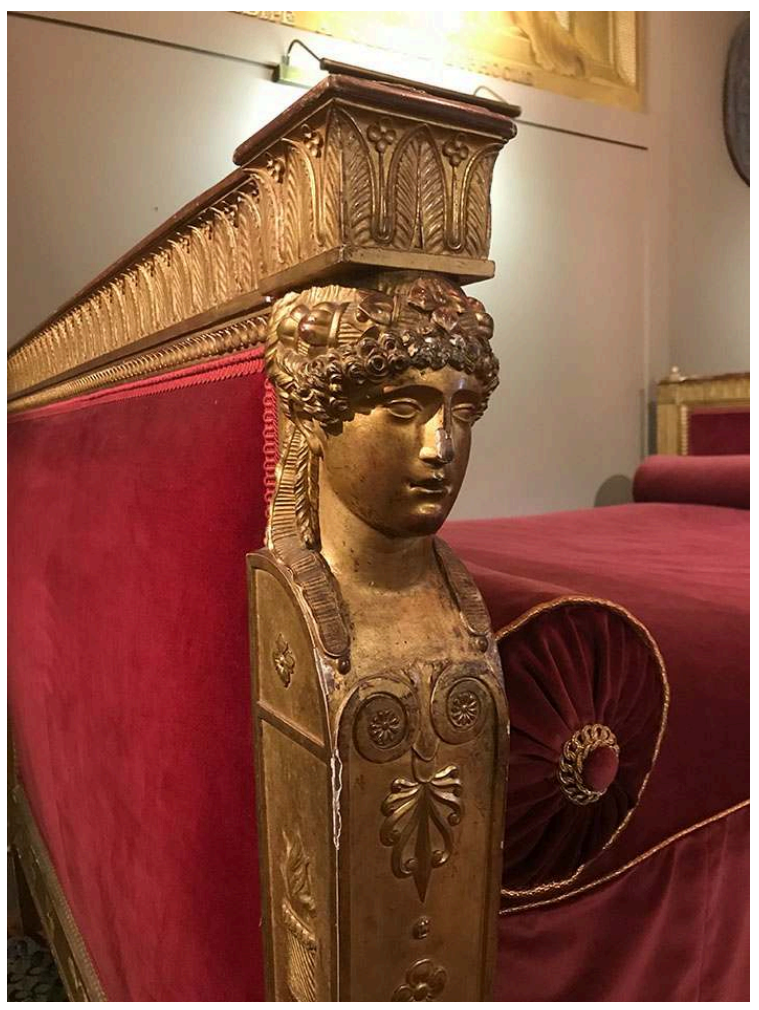

Détail de la figure en gaine du lit.

Phot. Renaud Serrette. () Renaud Serrette.

3 Le lit du CMN est très proche d'un lit aujourd'hui conservé au château de Fontainebleau (fig. 3). Ce lit fut réalisé vers 1799, vraisemblablement sur des dessins des architectes Charles Percier (1764-1838) et Pierre-François-Léonard Fontaine (1762-1853) pour l'ameublement des Directeurs au palais du Luxembourg. Il servit dans la chambre de Napoléon Bonaparte, alors consul, au palais des Tuileries, puis passa, en 1804, dans la chambre du pape Pie VII au pavillon de Flore. Il fut finalement envoyé en 1805 au château de Fontainebleau pour la chambre de l'Empereur au $1^{\text {er }}$ étage, où il servit jusqu'en 1807, puis fut placé dans la chambre de ses petits appartements du rez-dechaussée à partir de 1810 , où il se trouve toujours ${ }^{4}$. 


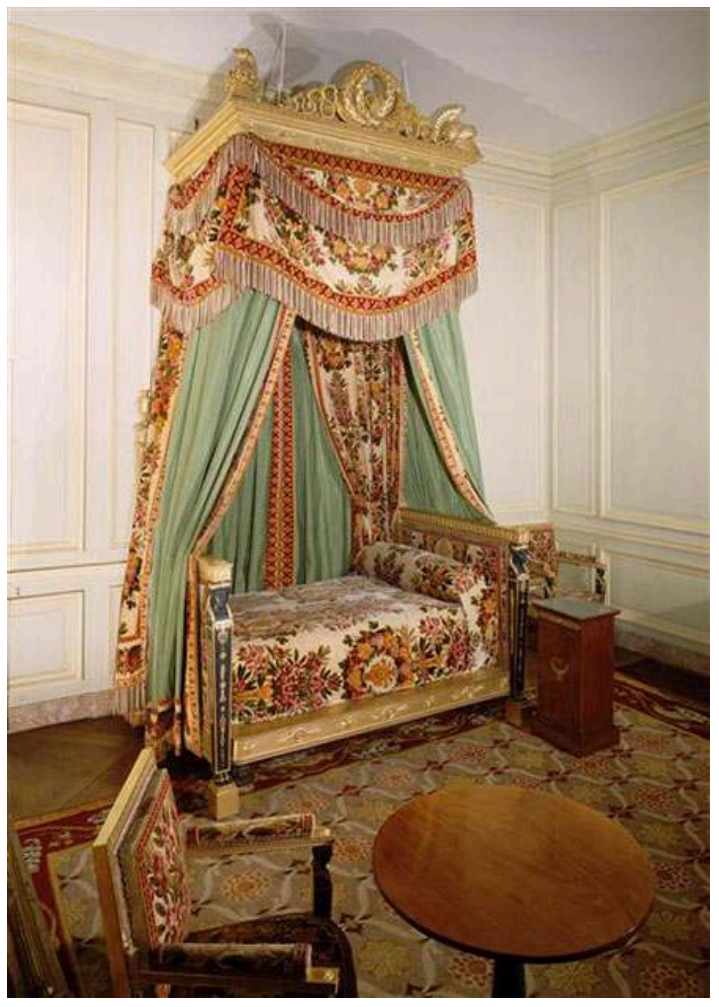

Attribué à Jacob Frères, lit de la chambre des petits appartements de l'Empereur à Fontainebleau, vers 1799, bois doré et bronzé, musée national du Château de Fontainebleau.

(c) RMN-Grand Palais, Château de Fontainebleau (n80-000784).

La comparaison des deux lits est instructive. Comme celui de Fontainebleau, le lit du $\mathrm{CMN}$ était à l'origine " en chaire à prêcher ${ }^{5}$ » avec des montants arrière originellement plus hauts que ceux avant. D'après les sources, son ciel de lit était très proche de celui de Fontainebleau ${ }^{6}$, avant que ce dernier ne soit enrichi en 1810 de casques, couronnes de laurier et rubans. Sa traverse avant simplement moulurée ne semble pas d'origine, celle de Fontainebleau est d'ailleurs cintrée, avec des ornements sculptés (patères, palmettes et étoiles). Un examen attentif montre encore que le lit du CMN a été élargi de quelques centimètres en longueur et en profondeur. Enfin, il présente aussi sous sa dorure actuelle la trace d'un réchampissage en bronze, comme c'est toujours le cas pour celui de Fontainebleau?.

5 Le lit de Fontainebleau est aujourd'hui attribué à l'entreprise Jacob Frères, qui fournissait la grande majorité des meubles de menuiserie commandés par le GardeMeuble. Sa similarité quasi parfaite avec le lit du CMN permettrait ainsi d'envisager cette attribution pour le lit retrouvé à Bouges.

\section{La chambre de Napoléon à Saint-Cloud}

Les différentes marques relevées sur le lit du CMN se rapportent non pas aux Tuileries mais au château de Saint-Cloud. Il porte en effet la marque au fer de ce château, apposée sous le Consulat, ainsi que le numéro «StC 120 » au pochoir, correspondant à l'inventaire de ce château en $1824^{8}$. 
Figure 4

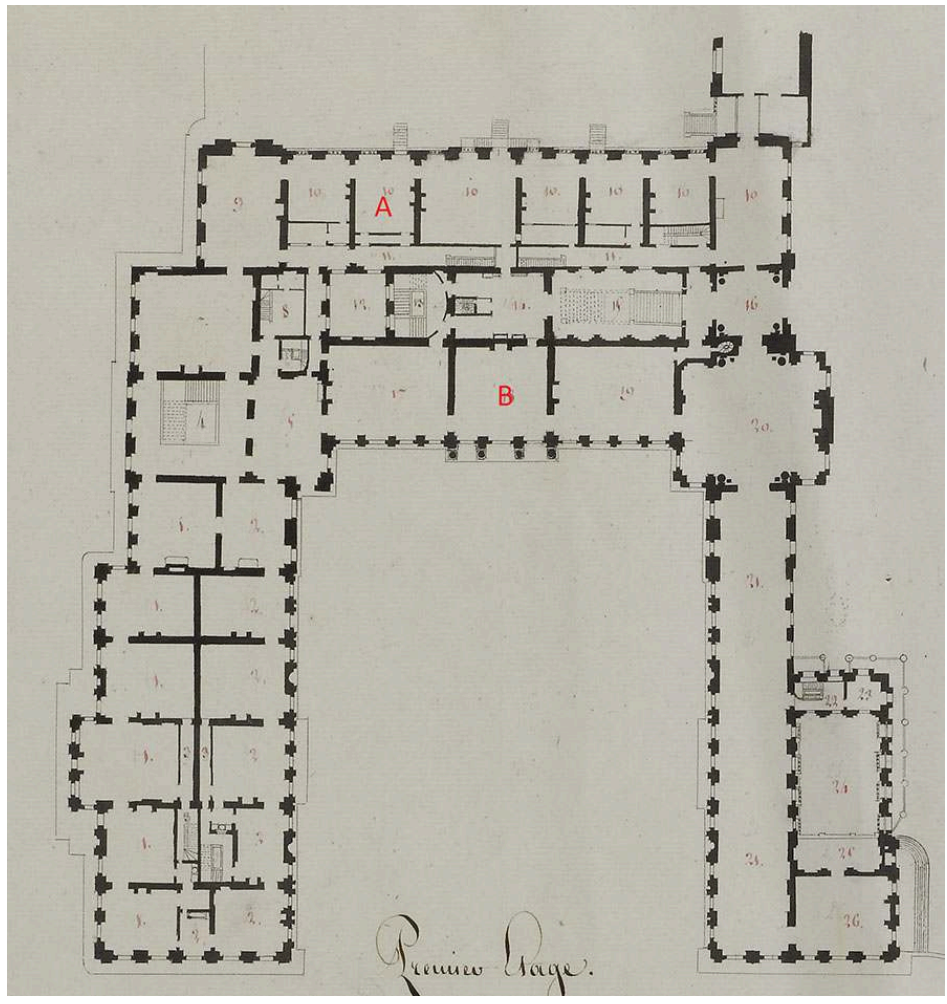

Anonyme, plan du premier étage du palais de Saint-Cloud, 1er quart du XIXe siècle, Mobilier national, inv. 120. A : chambre de l'appartement de l'Orangerie ; B : chambre provisoire du comte d'Artois puis chambre de représentation de Louis XVIII.

Phot. Patrick Cadet. (C) CMN, 2010 (nPCW10-0821).

7 Il était installé dans la chambre du consul Napoléon Bonaparte, devenu empereur en 1804. La pièce se trouvait dans l'appartement ouvrant de plain-pied sur le parterre de l'Orangerie (fig. 4). Les inventaires du château, dressés en l'an XIII (1804-1805) ${ }^{9}, 1807^{10}$ et $1812^{11}$ le décrivent ainsi reposant sur une estrade recouverte de drap cramoisi, le coucher et son ciel garnis de velours de soie vert à rayures satinées, brodé d'étoiles en or, et doublés de taffetas vert. Il était accompagné par quatre fauteuils ${ }^{12}$, une bergère et un canapé13 (fig. 5) en acajou aux pieds en griffes dorés, conservé de nos jours dans les collections du Mobilier national. Un lavabo, aujourd'hui au château de Fontainebleau ${ }^{14}$, et une commode en acajou à deux vantaux, ornée " de camées en bronze doré or mat " et d'un bas-relief avec une course de char ${ }^{15}$, complétaient l'ensemble. 


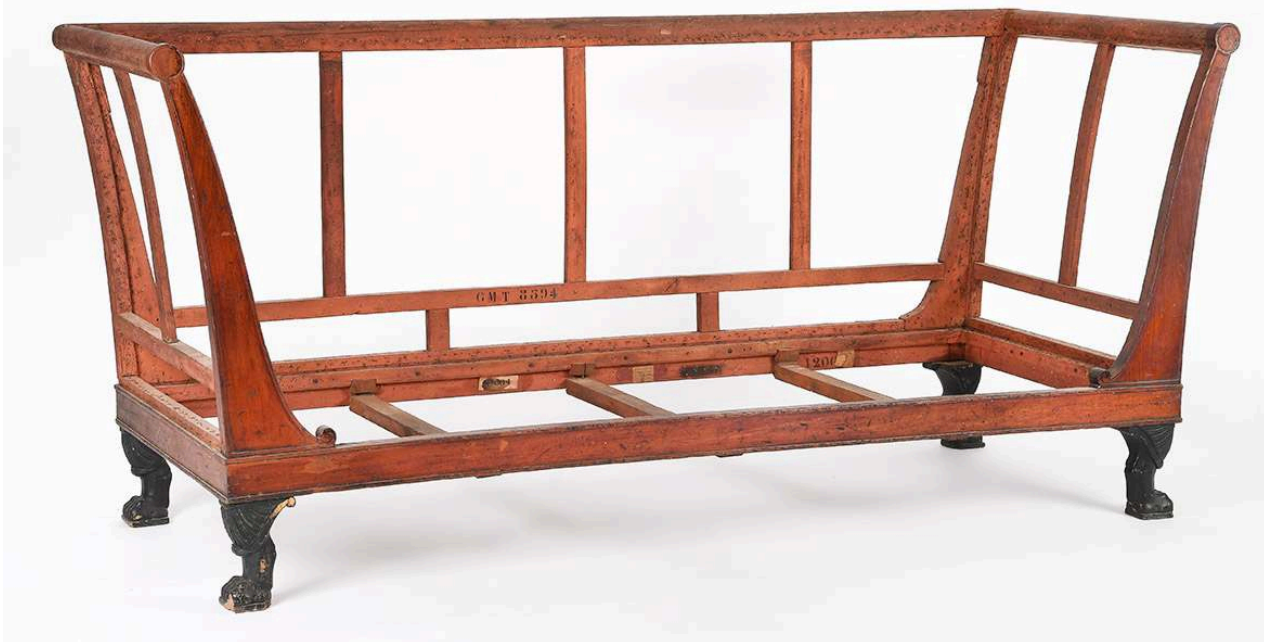

Par les frères Jacob, canapé de la chambre de l'Empereur à Saint-Cloud, 1802, Mobilier national, GMT 8594.

Phot. Isabelle Bideau. (c) Mobilier national, janvier 2019.

8 La pièce était encore décorée de plusieurs meubles et objets du XVIII ${ }^{\mathrm{e}}$ siècle. Derrière le lit étaient ainsi tendues une grande tapisserie tissée entre 1778 et 1792 aux Gobelins d'après un carton d'Antoine Coypel, Suzanne accusée par les vieillards ${ }^{16}$, et sur les murs latéraux, deux tapisseries d'après les Stanze (Vatican) de Raphaël, La Vision de la Croix (chambre de Constantin) et L'École d'Athènes (chambre de la Signature). Les deux bras de lumière en bronze doré à décor de tourterelles sur des nuages étaient ceux livrés par Lucien-François Feuchère (v. 1750-1828) pour le cabinet de toilette de Marie-Antoinette à Saint-Cloud en $1788^{17}$ (fig. 6). Quant aux chenets "surmontés d'un vase à guirlandes et têtes de béliers ", ils ne sont pas sans évoquer les créations de Quentin-Claude Pitoin (v. 1720-1777) sous Louis XVI ${ }^{18}$. 
Figure 6

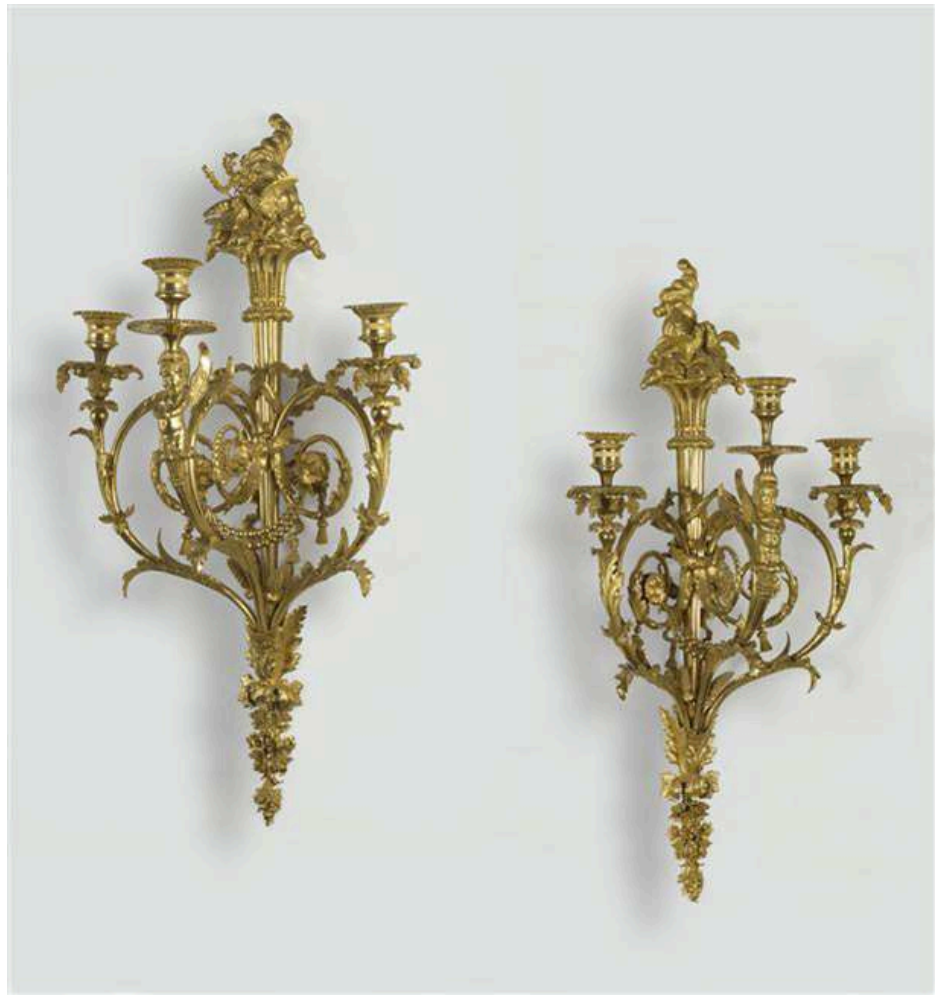

Lucien-François Feuchère, paire de bras de lumière de la chambre de l'Empereur à Saint-Cloud, 1788, bronze doré. Musée du Louvre, OA 5256 et 5257.

Phot. Martine Beck-Coppola. (C) RMN-Grand Palais, musée du Louvre (n07-537448).

9 Sur la cheminée trônait encore un buste de Jules César, avec une draperie en bronze rehaussée de dorure, et une tête en pierre de touche, travail italien du xviII ${ }^{\mathrm{e}}$ siècle qui indiquait assez l'ambition du maitre des lieux ${ }^{19}$ (fig. 7). Deux autres bustes en terre cuite, représentant les deux neveux de Napoléon ${ }^{20}$, étaient la seule note personnelle de la pièce. Ils disparaissent d'ailleurs dans l'inventaire de 1812, après la naissance du roi de Rome, au profit de deux sphères montées sur des supports en acajou (fig. 8). 
Figure 7

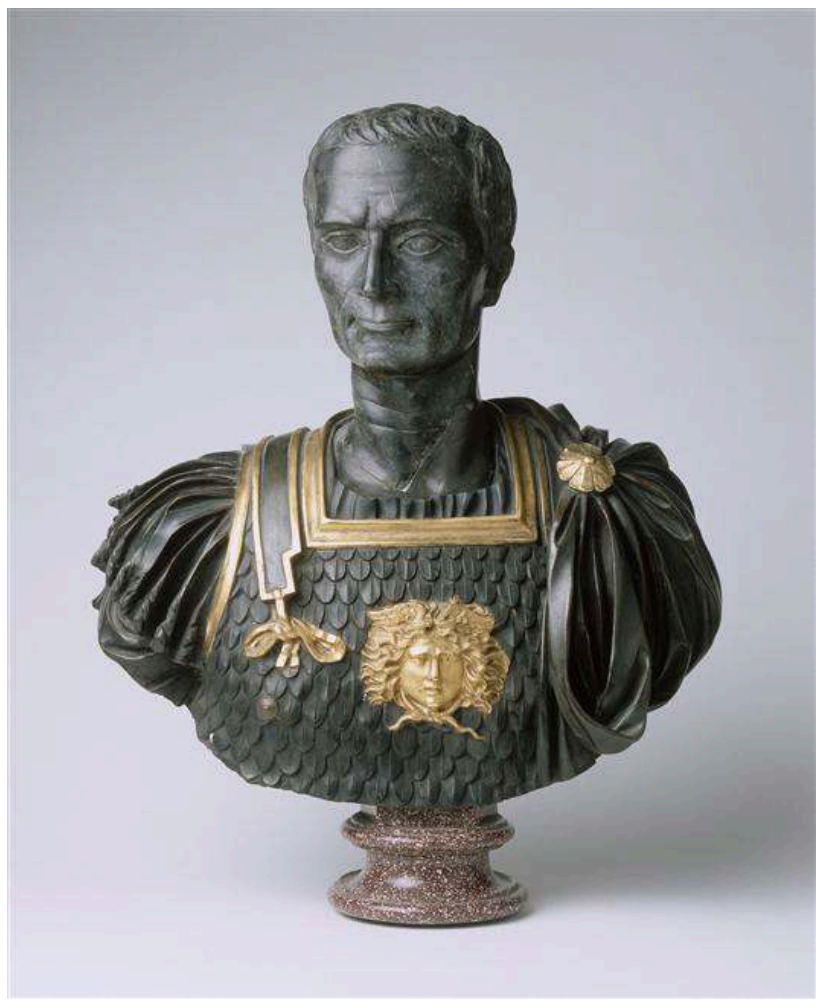

École italienne, buste de Jules César de la chambre de l'Empereur à Saint-Cloud, xvIII siècle, bronze, pierre de touche. Musée du Louvre, MR 3342.

Phot. Hervé Lewandowski. (c) RMN-Grand Palais, musée du Louvre (n98-005888).

Figure 8

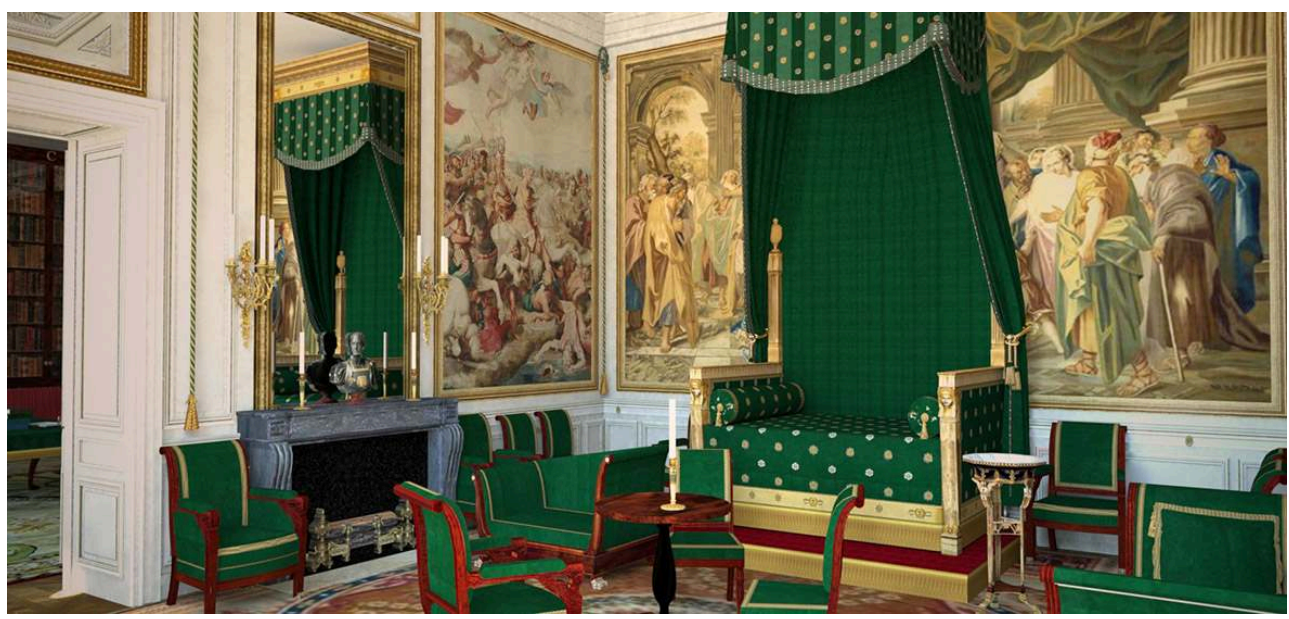

Restitution de la chambre de l'Empereur à Saint-Cloud (état 1812-1815).

Phot. Philippe Le Pareux. (c) Philippe Le Pareux, 2018.

\section{Commande ou remploi ?}

Le lit fut-il commandé pour l'installation du consul à Saint-Cloud en 1802 ? Le chantier de restauration du palais avait été confié en 1801 aux architectes Percier et Fontaine, 
qui supervisèrent également son remeublement. Peu de documents (devis, mémoires ou factures) nous sont parvenus sur cette opération d'envergure. Un rapport dressé en prairial an $\mathrm{XI}^{21}$ montre bien que les meubles furent majoritairement commandés aux frères Jacob, et une lettre adressée à Fontaine le 19 prairial an X (8 juin 1802) par le général Duroc, gouverneur du palais, semble indiquer qu'un lit neuf fut initialement envisagé par l'architecte : le Premier Consul «a appris avec peine ce qu'on lui a dit au sujet d'un lit que vous aviez commandé pour lui et qui, dit-on, doit coûter $24000 \mathrm{fr}$. Comme jamais vous ne m'en avez parlé, j'ai pensé que c'était un faux rapport. Le $1^{\mathrm{er}}$ Consul ne veut pas pour lui un lit plus cher que 1500 francs $^{22} »$.

11 De fait, plusieurs éléments plaident pour le remploi d'un lit sorti des réserves du GardeMeuble afin de suivre les recommandations de Bonaparte. Tout d'abord, il est probable que si les frères Jacob avaient reçu commande de l'intégralité des meubles en menuiserie de la pièce, ils auraient cherché à créer un ensemble homogène. Or le lit en bois doré diffère des sièges en acajou non seulement par ses matériaux mais aussi par son décor. De plus, le peu de temps laissé par Bonaparte à ses architectes pour remeubler le château ( 5 mois et demi) et ses consignes de remployer des meubles existants, par souci d'économie ${ }^{23}$, plaident en ce sens.

Il semble donc raisonnable d'avancer que le lit du CMN a probablement été réalisé par les frères Jacob vers 1799 et qu'il était destiné, comme son jumeau de Fontainebleau, à l'ameublement des Directeurs au palais du Luxembourg, avant d'être finalement envoyé à Saint-Cloud en 1802.

\section{Un lit de parade}

13 À cette date, le consul n'avait encore que deux résidences officielles à sa disposition : les Tuileries et Saint-Cloud. Les deux lits identiques établissaient ainsi une égalité protocolaire entre les deux demeures tout en préservant l'apparat nécessaire au pouvoir.

14 L'appartement de l'Orangerie était en effet un appartement de jour, uniquement destiné à la vie officielle de Napoléon. Pour son intimité, Napoléon disposait d'un appartement privé, comme aux Tuileries, à Fontainebleau et à Compiègne quelques années plus tard. D'abord situé au rez-de-chaussée de l'aile du fer à cheval, puis à partir de 1808, juste au-dessus de son appartement de l'Orangerie, ce petit appartement comptait une dizaine de pièces. Dans la chambre à coucher se trouvait un lit d'acajou dont la simplicité devait mieux correspondre aux goûts véritables de l'Empereur.

Dormant dans cette petite chambre, l'Empereur se réveillait habituellement vers $6 \mathrm{~h} 30$ $\mathrm{du}$ matin et descendait vers $9 \mathrm{~h}$ dans l'appartement sur l'Orangerie pour la cérémonie officielle du grand lever, qui lui permettait d'honorer certains courtisans et dignitaires ${ }^{24}$.

La chambre de l'Orangerie, avec son somptueux lit en bois doré, était donc avant tout une chambre de parade, destinée à manifester et célébrer la majesté et la personne impériale ${ }^{25}$. Cette symbolique devait d'ailleurs prendre toute sa mesure à la chute de l'Empereur.

Après la défaite de Waterloo (18 juin 1815), Saint-Cloud fut occupé par les soldats prussiens du feld-maréchal Gebhard Leberecht von Blücher (1742-1819). Selon une anecdote caricaturale publiée tardivement sous le Second Empire, ce dernier « prit un 
véritable plaisir à transformer cet admirable palais en un sale chenil ». Il se serait installé dans la chambre de l'Empereur, "suivi d'une meute de chiens qu'il faisait coucher sur les lits », tandis qu' " il se plaisait à souiller de ses bottes les draperies et les rideaux du lit de son ancien vainqueur. Il s'y vautrait tout habillé et déchirait les dentelles avec ses éperons ${ }^{26}$.»

\section{Une chambre pour le comte d'Artois}

18 À son retour d'exil, Louis XVIII prit possession de l'appartement de son prédécesseur, sur l'Orangerie. Le lit fut retiré, envoyé dans les magasins du Garde-Meuble de Paris et remplacé par un nouveau lit en bois doré, complété par de nouveaux sièges assortis en $1818^{27}$. Les autres membres de sa famille, son frère le comte d'Artois, son neveu et sa nièce le duc et la duchesse d'Angoulême, s'établirent dans l'aile du fer à cheval.

Le lit ne devait pas rester bien longtemps en réserve. Le 13 février 1820, le duc de Berry, autre neveu du roi, fut assassiné à la sortie de l'Opéra. Sa jeune veuve, Marie-Caroline de Bourbon-Sicile, enceinte de l'héritier du trône (futur comte de Chambord), ne souhaita pas retourner vivre seule à l'Élysée, sa résidence habituelle. Mme de Gontaut, gouvernante des Enfants de France, indique dans ses mémoires que son beau-père le comte d'Artois,

passant plusieurs heures près d'elle, parvint à la calmer, lui dit que le Roi mettait à son choix les résidences royales qui pouvaient lui convenir. Elle accepta pour le présent celle de Saint-Cloud et demanda d'y aller immédiatement avec sa fille. Ce même soir (le 14 février), elle y fut établie ainsi que Mademoiselle dans l'appartement de Monsieur le duc et Madame la duchesse d'Angoulême ${ }^{28}$.

Le Garde-Meuble dut prévoir un aménagement plus pérenne pour le séjour de la Cour à l'été suivant. Le comte d'Artois accepta alors de laisser à sa belle-fille son appartement du rez-de-chaussée de l'aile du fer à cheval pendant qu'il s'installait provisoirement dans les appartements d'apparat du premier étage sur la cour, composé de trois pièces principales.

21 Le premier salon, ancienne salle du Trône de Napoléon, devint ainsi son antichambre, qui ne fut pas modifiée. Le salon des Princes, au centre, fut transformé en chambre à coucher (voir fig. 4), et le salon cramoisi, qui terminait l'enfilade, en cabinet de travail. Si l'aménagement de ce dernier se fit simplement en ajoutant une bibliothèque et des éléments du surtout de Charles IV d'Espagne, celui de la chambre fut plus important. Le mobilier mis en place sous Napoléon fut entièrement retiré, à l'exception du lustre, du tapis de Beauvais à trophées militaires, des chenets, des deux flambeaux ${ }^{29}$ et des deux torchères en faïence de Sarreguemines imitant le porphyre ${ }^{30}$. Les deux bras de lumière de la cheminée, livrés pour cette pièce quand elle était le salon des jeux de MarieAntoinette ${ }^{31}$, furent aussi conservés, tout comme ceux provenant du cabinet de travail de Louis XVI à Saint-Cloud ${ }^{32}$, placés sur les murs latéraux.

Le tapissier Lejeune tendit la pièce d'une soierie en cannetillé bleu à rosettes d'or ${ }^{33}$, avec une bordure en brocart bleu et or tissé en 1811-1812 pour Versailles, dont des échantillons sont toujours conservés au Mobilier national ${ }^{34}$. Le Garde-Meuble proposa en juillet 1820 de remployer ici le lit de la chambre de l'Empereur, qui revint ainsi à Saint-Cloud cinq ans après son départ :

«État des objets provenant du dépècement de divers meubles...

2807. Une couchette en chaire à prêcher bois doré, pieds à socles, montants de 
devant à cariatides, ceux de derrière surmontés de vases forme d'urnes, dossiers

barrés garnis en crin et couverts en toile douce... 300 francs $^{35}$. "

Le tapissier Lejeune fut chargé de le redorer entièrement et de le recouvrir du même cannetillé et du même brocart, avec une riche passementerie en or. Son devis fut validé le 10 août $1820^{36}$ et les travaux achevés en 1821. Le lit fut placé devant un trumeau de glace, probablement celui faisant face à l'entrée depuis l'antichambre ${ }^{37}$. Deux canapés, six fauteuils et douze chaises en bois doré à dossiers en crosse, accompagnés de deux tabourets de pied et un écran de cheminée, furent également prélevés dans les réserves du Garde-Meuble et recouverts du même cannetillé bleu pour accompagner le lit. Ces sièges provenaient $\mathrm{du}$ premier salon de l'Empereur aux Tuileries, où ils étaient recouverts de damas cramoisi avec bordures en brocart. Retirés en août 1819, ils furent envoyés à Saint-Cloud dès 1820, et restèrent dans la même pièce jusqu'en 1869, après avoir été regarnis en 1839 de tapisseries de Beauvais. Ils sont aujourd'hui conservés au Mobilier national $^{38}$ (fig. 9).

Figure 9

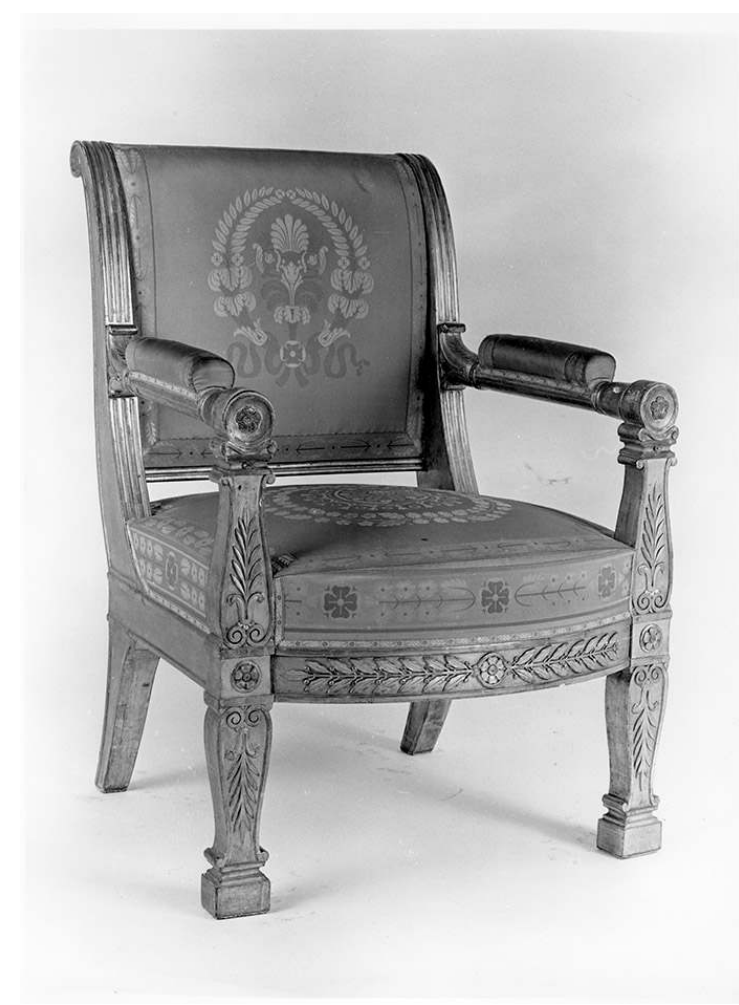

Jacob-Desmalter, fauteuil de la chambre de représentation de Louis XVIII à Saint-Cloud, 1809, bois doré. Mobilier national, GMT 8531, 8532, 1534-1/2.

(c) Mobilier national.

La commode de la chambre de l'impératrice à Saint-Cloud, réalisée par Jacob-Desmalter en 1803-1804 ${ }^{39}$, fut installée en face du lit, et s'accompagnait d'un bureau plat en acajou. Un somno (table de chevet) en acajou à décor de branches de lys en bronze doré, un bidet, un pot à eau et un pot de nuit en porcelaine de Sèvres furent eux aussi sortis des réserves $\mathrm{du}$ Garde-Meuble ${ }^{40}$. Une pendule en bronze doré représentant Lucrèce ${ }^{41}$ ornait le manteau de cheminée, et une réduction en bronze du Moïse de Michel-Ange, aujourd'hui conservée au château de Versailles ${ }^{42}$, devait prendre place sur la commode. 

conservée. Quelques compléments furent apportés à son aménagement : la commode fut remplacée par une autre à socle plein en acajou et à trois vantaux d'époque Empire, tandis que deux vases en porcelaine de Sèvres, de forme Médicis et à décor de fleurs et de papillons sur fond blanc étaient ajoutés ${ }^{44}$. Enfin, en 1824, quatre grands tableaux de paysages par Claude-Joseph Vernet, commandés en 1767 par le marquis Jean-Joseph de Laborde pour son château de La Ferté-Vidame (Eure-et-Loir) ${ }^{45}$, furent accrochés sur la soierie bleue et or (fig. 10).

Figure 10

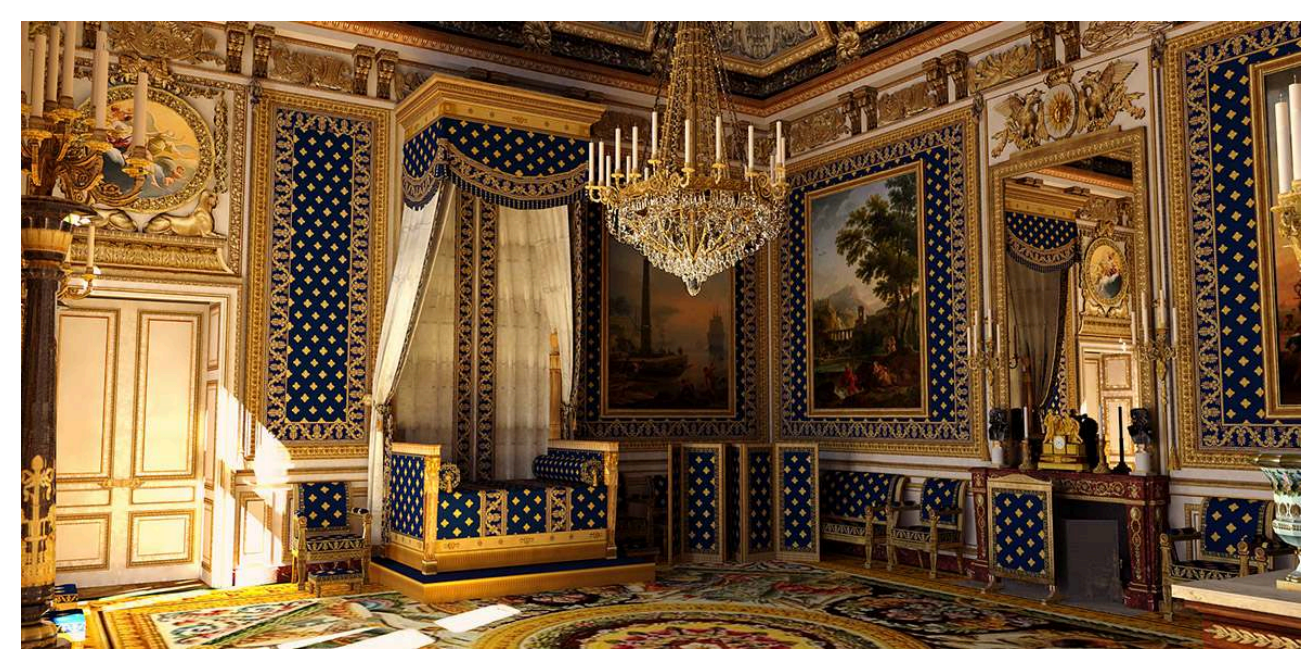

Restitution de la chambre de représentation de Louis XVIII à Saint-Cloud (état 1824-1825).

Phot. Philippe Le Pareux. (c) Philippe Le Pareux, 2018.

L'inventaire de 1824 désigne de fait la pièce comme la "chambre de représentation " du souverain, située au cœur des appartements de réception. La pièce était déjà au XVII ${ }^{\mathrm{e}}$ siècle la chambre du roi, occupée par Louis XIV lors de ses séjours à Saint-Cloud. Son emplacement au centre de la façade du château, côté cour, n'était pas sans évoquer la 
chambre de parade du roi à Versailles avant la Révolution, qu'avaient bien connue Louis XVIII et le comte d'Artois.

On sait que les Bourbons restaurés accordèrent, contrairement à Napoléon, moins d'importance aux salles du Trône qu'aux chambres royales, dont la symbolique monarchique était plus traditionnelle. Aux Tuileries, les efforts du Garde-Meuble s'étaient ainsi portés dès 1817 sur le renouvellement des somptueuses garnitures textiles de la chambre du roi, aujourd'hui conservées au musée du Louvre ${ }^{46}$, alors que le nouvel ameublement de la salle du Trône ne fut commandé qu'en 1818, et installé en 1822 seulement.

30 À Saint-Cloud, la pérennisation de la chambre de représentation en 1821 suivait donc le programme politique établi par la monarchie restaurée. Mais sa symbolique était encore plus forte. En effet, aux Tuileries comme à Compiègne ou Fontainebleau, la suppression des petits appartements avait impliqué la transformation des chambres officielles en chambres véritables du souverain, où il dormait réellement. À SaintCloud, Louis XVIII renonça aussi au petit appartement du second étage. La création d'une chambre dans les grands appartements du château lui permettait ainsi opportunément de transformer l'appartement ouvrant sur le parterre de l'Orangerie en appartement privé, tandis que la chambre côté cour devenait une chambre officielle. Non seulement le roi n'y dormait pas, mais encore la pièce jouait seule le rôle de représentation du pouvoir, la salle du Trône de Napoléon étant supprimée.

Cette véritable chambre de parade dans son acception de l'Ancien Régime est ainsi à notre connaissance unique sous la Restauration ${ }^{47}$.

Son aménagement est aussi paradoxal. Alors que la pièce retrouvait un usage rappelant le XVIII ${ }^{\mathrm{e}}$ siècle, l'administration préféra utiliser des meubles modernes plutôt que de conserver et renforcer l'ameublement installé sous l'Empire et qui faisait la part belle à de beaux meubles d'Ancien Régime: la tenture et la garniture des sièges n'étaient autres que le meuble d'été de Marie-Antoinette à Versailles, tandis que les deux commodes provenaient de son salon des jeux à Compiègne. Le paravent et les pliants avaient été réalisés pour la chambre et le cabinet du conseil de Louis XVI à Compiègne et la pendule provenait de sa pièce de la Vaisselle d'or à Versailles ${ }^{48}$. Il est probable que Louis XVIII et le comte d'Artois aient reconnu ces meubles qu'ils avaient vus avant de partir en exil. Même le buste en bronze du Grand Condé, que les Bourbons avaient fait placer à leur retour, fut retiréé ${ }^{4}$. Il semble ainsi que le symbolisme de la pièce était assez fort pour qu'il ne soit pas nécessaire d'y rassembler des meubles de provenance royale ou de rappeler les grandes figures du passé.

On remarquera cependant que l'appellation « chambre de représentation", préférée à celle de "chambre de parade", dénote un manque d'assurance et ne semble pas s'assumer. Les Bourbons durent rapidement sentir l'incongruité d'une pièce avec une symbolique d'un autre temps.

\section{Les tribulations des $\mathrm{XIX}^{\mathrm{e}}$ et $\mathrm{XX} \mathrm{X}^{\mathrm{e}}$ siècle}

34 À la mort de Louis XVIII, en 1824, son frère le comte d'Artois lui succéda sous le nom de Charles X. Sa belle-fille, Marie-Thérèse, duchesse d'Angoulême, devint dauphine. Habitant l'appartement mitoyen dans l'aile du fer à cheval, elle souhaita rapidement pouvoir tenir sa cour le soir dans les salons de réception voisins. En 1825, le concierge 
de Saint-Cloud écrivait ainsi au baron Armand Thierry de Ville d'Avray, intendant du Garde-Meuble :

Par ordre de Madame la Dauphine, Monsieur le vicomte d'Agoult gouverneur du château m'a chargé de vous prévenir que les grands appartements sont destinés à l'avenir aux réceptions de Madame la Dauphine. Cette nouvelle destination nécessitera l'enlèvement $\mathrm{du}$ lit $\mathrm{du}$ salon bleu et de la commode qui y existe, la confection de chaises et fauteuils à porter au meuble pour augmenter l'ameublement de ce salon ${ }^{50}$.

Le 18 août 1825, le lit fut envoyé avec ses étoffes dans les magasins du Garde-Meuble à Paris $^{51}$. Si l'estrade est vendue en $1829^{52}$, le lit figure encore sur les inventaires du Garde-Meuble en $1833^{53}$. On perd ensuite sa trace. Il fut probablement vendu par l'administration dans la $2^{\mathrm{e}}$ moitié $\mathrm{du} \mathrm{XIX}^{\mathrm{e}}$ siècle. Une étiquette de transport de la Compagnie des chemins de fer de l'Ouest atteste son passage au Mans entre 1855 et 1909. Il réapparaît dans les années 1960 , recouvert de velours de soie rouge, dans la chambre à coucher de Juan de Beistegui (1930-2017), dans son hôtel particulier de la rue de Varenne (Paris, VII ${ }^{\mathrm{e}}$ arr.). L'éminent collectionneur savait qu'il avait pu servir à l'empereur Napoléon ${ }^{54}$. Il y fut remarqué par François Macé de Lépinay, inspecteur des Monuments historiques, avant que Juan de Beistegui ne s'en sépare dans les années 1970 et qu'il ne réapparaisse à Drouot en 1983.

Le Centre des monuments nationaux a aujourd'hui entrepris une étude approfondie de la menuiserie et de la dorure de ce lit afin de le restaurer et de le présenter au musée historique du Domaine national de Saint-Cloud, dont il a la gestion. Une étude textile est également prévue afin d'établir les bases scientifiques d'une restitution de sa garniture selon l'état Empire.

Sa présentation constituera un exceptionnel témoignage de l'ameublement du château dans la $1^{\text {re }}$ moitié du xIX ${ }^{e}$ siècle, et le signe tangible de la majesté impériale puis royale.

\section{NOTES}

1. - Vente Paris, hôtel Drouot, étude Couturier et Nicolay, 16 juin 1983, lot 82.

2. - Inv. BOU1983101619. On envisagea un moment comme lieu de dépôt les châteaux de Carrouges (Orne) et de Maisons-Laffitte (Yvelines), sans suite. Aucun document consulté (archives du CMN, archives du bureau de la Conservation du patrimoine mobilier et instrumental du ministère de la Culture et archives de la Médiathèque de l'architecture et du patrimoine) ne donne les motifs de cet achat.

3. - Ces vases n'ont pas été retrouvés au château de Bouges. Il est possible qu'ils aient été cassés et perdus lorsque le lit fut stocké pendant plus d'un an après sa vente dans les entrepôts de l'hôtel Drouot. Les vis et les roulettes sont également manquantes.

4. - SAMOYAULT, Jean-Pierre, SAMOYAULT-VERLET, Colombe. Guide du musée national du Château de Fontainebleau. Paris : RMN, 2002, p. 140 ; Id. Mobilier français Consulat et Empire. Paris : GourcuffGradenigo, 2009, p. 76 ; VITTET, Jean. «L'appartement de Napoléon I ${ }^{\text {er }}$ à Fontainebleau, histoire et métamorphoses ». Dossier des Amis du château de Fontainebleau, nº 15, 2018, p. 6. 
5. - Le lit en chaire à prêcher est apparu à la fin du XVIII ${ }^{e}$ siècle. Il se caractérise par un coucher parallèle au mur et est surmonté par un ciel plus petit supporté par deux colonnes ou structures métalliques placées à l'aplomb des montants arrière.

6. - Une description de ce ciel en 1824 (Archives nationales, désormais abrégé $\mathrm{AN}, \mathrm{O}^{3} 2076$ ) correspond en effet à l'aspect du ciel de Fontainebleau. Ce dernier fut pourtant créé en 1804 par Jacob-Desmalter. Le ciel de lit de Fontainebleau copiait-il celui de Saint-Cloud?

7. - Atelier de la feuille d'or, DUBOST, Marie et Florent. «Étude du lit de Napoléon à Fontainebleau, comparatif avec le lit de Napoléon de Saint-Cloud / Étude du décor doré du lit de Napoléon à Saint-Cloud", 2018 et PERFETTINI, Jean. «Étude du lit de la chambre de l'Empereur Napoléon I Ir au château de SaintCloud». Support-bois, 2018.

8. - Il porte encore les marques au pochoir N508 (numéro des magasins du Garde-Meuble avant 1820), 24092 (numéro de rentrée au Garde-Meuble en 1825), 2160 (numéro du Garde-Meuble en 1833) et 332 (numéro non identifié).

9. - AN, $\mathrm{O}^{2} 730$.

10. - AN, $\mathrm{O}^{2} 731$.

11. - AN, $\mathrm{O}^{2} 740$.

12. - Les sièges actuellement présentés dans la chambre de l'Empereur au château de La Malmaison (GMT 1536, M.M.40.47.2102), bien que portant l'étiquette «chambre à coucher du consul », furent en fait placés dès la livraison dans un appartement de prince. Voir SAMOYAULT, Jean-Pierre. «Le cadre de vie de Napoléon Bonaparte». Dans JUVIGNY, Sophie (dir.). Napoléon Bonaparte à Saint-Cloud. Du coup d'État de Brumaire à la fin de l'Empire. Cat. exp., musée municipal de Saint-Cloud, 10 nov. 1999-30 janv. 2000. Saint-Cloud : musée municipal, p. 83-84, nº 119.

13. - GMT 8594 ; voir GAUTIER, Jean-Jacques (dir.). Sièges en société. Histoire du siège du Roi-Soleil à Marianne. Cat. exp., Paris, galerie des Gobelins, 25 avril-24 sept. 2017. Paris : Gourcuff-Gradenigo, 2017, p. 184.

14. - SAMOYAULT, Jean-Pierre, SAMOYAULT-VERLET, Colombe. Le Mobilier du général Moreau : un ameublement à la mode en 1802. Cat.exp., musée national du Château de Fontainebleau, 16 juin-14 sept. 1992. Paris : Réunion des musées nationaux, 1992, p. 60.

15. - Une commode très proche, à l'exception du décor des vantaux, est conservée au château de Fontainebleau (inv. F75c).

16. - Issue de la $6^{\mathrm{e}}$ tenture de l'Ancien Testament, elle fut restaurée par le rentrayeur Bellanger en 1801 pour être installée à Saint-Cloud (AN, $\left.\mathrm{O}^{2} 875\right)$.

17. - Musée du Louvre, OA 5256 et 5257.

18. - Voir des chenets proches conservés au château de Versailles (OA 5177).

19. - Musée du Louvre, inv. MR 3342. Voir BRESC-BAUTIER, Geneviève (dir.). Les Sculptures européennes du musée du Louvre, Moyen Âge, Renaissance et Temps modernes. Paris : Musée du Louvre/ Somogy, 2006, p. 407.

20. - Il s'agissait des fils de Caroline Murat, Achille (1801-1847) et Lucien (1803-1878).

21. - Bibliothèque nationale de France, $\mathrm{ms} 6583$, fo 168.

22. - COURAL, Jean. Soieries Empire. Mobilier national. Paris : RMN, t. XXV, 1980, p. 13 ; VITTET, Jean. Dans les rêves de Napoléon, la première chambre de l'Empereur à Fontainebleau. Dijon: Faton, 2016, p. 56. Je remercie Jean Vittet de m'avoir signalé cette citation.

23. - SAMOYAULT, Jean-Pierre. «Les remplois de sculptures et d'objets d'art dans la décoration et l'ameublement du palais de Saint-Cloud sous le consulat et au début de l'Empire ». Bulletin de la Société de l'histoire de l'art français, 1971, p. 154.

24. - De tels grands levers à Saint-Cloud sont mentionnés les 3 octobre 1802, 15 août 1806 et 7 mai 1811 (SHUERMANS, Albert. Itinéraire général de Napoléon I ${ }^{\text {er }}$ ( ${ }^{\mathrm{e}}$ éd.). Paris : Jouve, 1911, p. VIII, 157, 230 et 37$)$. 
25. - Notons qu'une autre chambre de parade fut envisagée en 1804 côté cour, dans la salle du Conseil d'État. Ce projet resta sans suite et la pièce devint finalement la salle du Trône (COURAL, Jean. Op. cit., p. 93-94).

26. - SAINT-ALBIN, Philippe de, et DURANTIN, Armand. Le Palais de Saint-Cloud, résidence impériale. Paris : Librairie Centrale, 1864, p. 218.

27. - La date précise du départ du lit n'est pas documentée. Nous avons pu identifier le nouveau lit dans les réserves du mobilier national (GME 1478). Les sièges livrés en 1818 sont aujourd'hui conservés au château de Fontainebleau (salon du prince impérial dans l'appartement des Chasses).

28. - GONTAUT-BIRON, Joséphine de Montaut-Navailles, duchesse de. Mémoires de Mme la duchesse de Gontaut, gouvernante des Enfants de France pendant la Restauration. Paris : Plon-Nourrit et Cie, 1893, p. 211.

29. - Des flambeaux du même modèle sont conservés au château de Fontainebleau (F3565). DUMONTHIER, Ernest. Les Bronzes du Mobilier national. Paris : C. Massin, 1911 et SAMOYAULT, JeanPierre. Pendules et bronzes d'ameublement entrés sous le Premier Empire. Paris: RMN, 1989, p. 201, nº 189. Je remercie Jean Vittet, conservateur au château de Fontainebleau, et Mme Emmanuelle Federspiel, inspectrice au Mobilier national, pour leur aide sur ces flambeaux.

30. - Installées à partir de 1818 seulement dans la pièce (AN, $\left.\mathrm{O}^{3} 2075\right)$, elles sont conservées au Mobilier national (GML 6327-1/2). Voir DUPUY-BAYLET, Marie-France. Les Bronzes du Mobilier national, 1800-1870. L'heure, le feu, la lumière. Cat. exp., Paris, galerie nationale des Gobelins, 21 sept. 2010-27 février 2011. Dijon : Faton, 2010, p. 46-47, nº 11.

31. - Conservée au château de Versailles, OA 5250 ou 5251.

32. - Mobilier national, GML 3806 et 6238.

33. - Des lés de ce cannetillé ont été identifiés à l'occasion de cette étude dans les réserves du Mobilier national (GMMP 92) par Marie-Amélie Tharaud, que je remercie vivement. Cette soierie avait été livrée par le soyeux Jean-Pierre Séguin.

34. - COURAL, Jean. Op. cit., p. 75-78, $\mathrm{n}^{\circ} 11$.

35. - AN, $\mathrm{O}^{3} 2065$.

36. - Id.

37. - De fait, une paire de bras de lumière fut enlevée et renvoyée au Garde-Meuble (AN, $\mathrm{O}^{3} 2065$ ).

38. - Notamment sous les numéros GMT 8531-1/2, 8532-1 à 6, 1534-1/2 (communication de JeanJacques Gautier que je remercie). Les sièges sont bien visibles sur les photographies de la pièce prises en 1868-1871 par Armand Schneider (Bibliothèque historique de la Ville de Paris).

39. - Non localisée mais identique à une commode conservée au Mobilier national, en dépôt au château de Fontainebleau (GME 10688/F4064). SAMOYAULT, Jean-Pierre. Musée national du château de Fontainebleau. Meubles entrés sous le Premier Empire. Paris: RMN, 2004, n 314, p. 373-374.

40. - AN, $\mathrm{O}^{3} 2065$ et 2075.

41. - Cette pendule provenait du salon cramoisi voisin et fut échangée avec la pendule du salon des Princes. Sa localisation actuelle est inconnue.

42. - MV 7757.

43. - $\mathrm{AN}, \mathrm{O}^{3} 2075$.

44. - $\mathrm{AN}, \mathrm{O}^{3} 2075, \mathrm{n}^{\circ} 8083$ et $\mathrm{O}^{3} 2065, \mathrm{n}^{\circ} 5779$.

45. - Aujourd'hui à Avignon, au musée Calvet.

46. - SAMOYAULT-VERLET, Colombe. «L'ameublement des châteaux royaux à l'époque de la Restauration ». Dans Unâge d'or des arts décoratifs (1814-1848). Cat. exp., Paris, Grand Palais, 10 oct.-30 déc. 1991. Paris : RMN, 1991, p. 42-48.

47. - On ne sait en revanche si elle fut utilisée par Louis XVIII.

48. - Les sièges, dont une partie a conservé sa garniture versaillaise, sont conservés à Fontainebleau (notamment $\mathrm{F}$ 3436). Les commodes sont conservées à Compiègne (OA 5442 et 
5443). Le paravent est au château de Versailles (OA 9395). Les pliants et la pendule ne sont plus localisés. Signalons que dans l'ancienne salle du Trône voisine figuraient encore quatre torchères de la galerie des Glaces de Versailles (non localisées).

49. - Musée du Louvre, MR 3343.

50. - $\mathrm{AN}, \mathrm{O}^{3} 2066$, lettre du 20 juin 1825.

51. - $\mathrm{AN}, \mathrm{AJ}^{19} 300$, fo $25, \mathrm{n}^{\circ} 120$.

52. - $\mathrm{AN}, \mathrm{AJ}^{19} 619, \mathrm{f}^{\circ} 77$.

53. - AN, $\mathrm{AJ}^{19} 625$, vol. 2 , fo $56, \mathrm{n}^{\circ} 2160$.

54. - Je remercie Isabelle de Gourcuff, sa fille, pour ces précisions.

\section{RÉSUMÉS}

L'identification récente dans les réserves du Centre des monuments nationaux d'un lit en bois doré d'époque Consulat a fourni l'occasion de retracer son histoire. Ce lit a probablement été réalisé par les frères Jacob vers 1799 pour les Directeurs au Luxembourg, avec un jumeau aujourd'hui conservé dans la chambre des petits appartements de l'Empereur à Fontainebleau. Le lit fut remployé en 1802 dans la chambre du Consul puis de l'Empereur Napoléon $\mathrm{I}^{\mathrm{er}}$ au château de Saint-Cloud, dans son appartement ouvrant sur l'Orangerie. Renvoyé au Garde-Meuble vers 1815 , le lit fut à nouveau réutilisé en 1820 à Saint-Cloud pour composer une chambre provisoire destinée au comte d'Artois, pièce qui devint l'année suivante la chambre de représentation de Louis XVIII. Cette chambre, dont l'existence était jusqu'à présent inédite, est une tentative insoupçonnée de la Restauration de rétablir l'usage des chambres de parade de l'Ancien Régime. Son usage désuet dans la France du XIX siècle n'eut pas de suite, et la pièce redevint un salon de réception dès 1825 . Renvoyé au Garde-Meuble, le lit fut probablement vendu dans la seconde moitié du XIX siècle, avant d'être racheté en 1983 par le Centre des monuments nationaux.

The recent identification in the reserve collections of the national monuments centre (Centre des monuments nationaux) of a gilded wooden bed of the Consulate period has offered the opportunity to look more closely at its history. The bed was probably made by the Jacob brothers in about 1799 for the Directors at the Luxembourg palace, with an identical bed which is held today in the bed chamber of the Emperor's small apartments at the palace of Fontainebleau. The bed was installed, in 1802, in the Consul's bed chamber at the palace of Saint-Cloud and remained in the apartments that open onto the Orangery when the Consul became the Emperor Napoleon 1. The bed was sent back to the state's furniture repository, the Garde-Meuble, in 1815 but was brought out again in 1820, at Saint-Cloud, in order to compose a provisional bed chamber for the Comte d'Artois. The following year, this room became King Louis XVIII's state reception bed chamber. This type of royal bedroom, not seen since the Revolution, was an unsuspected attempt by the restored Bourbon monarchy to re-establish the practice of state bedrooms of the Ancien Régime. But the function was too outdated at the beginning of the nineteenth century and the bedroom was transformed into a reception room from 1825 . The bed was sent back to the GardeMeuble and was then sold, probably during the second half of the nineteenth century. It was purchased by the Centre des Monuments nationaux in 1983. 
INDEX

Mots-clés : art décoratif, chambre, lit, Napoléon Ier, Louis XVIII, Charles-Philippe de France, comte d'Artois, Jacob Frères, Pierre-Léonard Fontaine, Charles Percier, Saint-Cloud, Fontainebleau, Centre des monuments nationaux

Keywords : decorative art, bedroom, bed, Napoleon, Louis XVIII, Charles-Philippe de France, Comte d'Artois, Jacob Brothers, Pierre-Léonard Fontaine, Charles Percier, Saint-Cloud, Fontainebleau, Centre des Monuments nationaux

\section{AUTEUR}

\section{RENAUD SERRETTE}

Référent collections (conservation, étude et présentation des collections), Centre des monuments nationaux renaud.serrette@monuments-nationaux 